\title{
Efficacy of moderate-intensity walking provided feedback by ECE PED0 on abdominal fat in overweight and obese women: A randomized, exercise study
}

\author{
Esra Göçer , Füsun Ardıç ${ }^{1}$ Nuray Akkaya ${ }^{1}$, Duygu Herek² \\ ${ }^{1}$ Department of Physical Medicine and Rehabilitation, University of Pamukkale, Faculty of Medicine, Denizli, Turkey \\ ${ }^{2}$ Department of Radiology, University of Pamukkale, Faculty of Medicine, Denizli, Turkey
}

Received: July 2017 Accepted: October 2017

\begin{abstract}
Objectives: The aim of this study was to investigate the effectiveness of walking with Equipment for Clever Exercise (ECE PEDO) compared to supervised, moderate-intensity, aerobic, treadmill walking exercise in overweight and obese women.

Patients and methods: Between September 2014 and January 2015, 28 women with overweight and obesity were assigned to two groups: supervised treadmill walking (Group 1, n=14) or walking with ECE PEDO (Group 2, n=14). The target heart rate (HR) corresponding to values of 50 to $70 \% \mathrm{VO}_{2} \max$ were determined by submaximal treadmill test. Group 1 was instructed walking at their target HR on treadmill. The number of steps in a min corresponding to the target $\mathrm{HR}$ was calculated by a criterion pedometer and Group 2 was instructed walking in this step range recorded to the ECE PEDO giving audible feedback. Before and after a 12-week exercise program, all participants were evaluated by Body Mass Index (BMI) and waist circumference (WC). Ultrasonographic visceral fat thickness (VFT) and ergospirometric $\mathrm{VO}_{2} \mathrm{max}$.

Results: The VFT decreased in both walking groups in association with reduced WC and weight loss and also significant increases in the $\mathrm{VO}_{2}$ max after exercise intervention $(\mathrm{p}<0.05)$. The VFT decreased only in women by walking with ECE PEDO compared to the other group $(\mathrm{p}<0.05)$.
\end{abstract}

Conclusion: Our study results showed that both moderate-intensity exercise provided by a novel pedometer and supervised treadmill walking showed significant improvements in abdominal obesity and $\mathrm{VO}_{2}$ max.

Keywords: Abdominal obesity; aerobic exercise; pedometer.

Overweight and obesity are described as abnormal or excessive fat accumulation which may impair health according to the definitions of the World Health Organization. ${ }^{[1]}$ Individuals with obesity have an increased risk of associated multiple health problems, including type 2 diabetes mellitus, hypertension, degenerative joint disease, dyslipidemia, and certain types of malignancies. ${ }^{[2]}$ The excess accumulation of abdominal fat (abdominal adiposity) is more associated with complications of the obesity than the excess total body fat. ${ }^{[3]}$

The addition of an exercise training program is a helpful strategy for the management of obesity and related complications. Current physical activity and exercise recommendations offer that the inclusion of aerobic exercise is essential for exercise programs for overweight/obesity management. ${ }^{[4]}$ The American College of Sports Medicine (ACSM) suggests 150 to $250 \mathrm{~min} /$ week of aerobic physical activity with moderate-intensity for individuals with overweight and obese..$^{[5]}$

Although physical activity is commonly recommended to reduce overall obesity, the effect of exercise-induced weight loss on abdominal adiposity has been still investigated. ${ }^{[6]}$ The direct relationship between the exercise intensity and a decrease in the

Corresponding author: Füsun Ardıç, MD. Pamukkale Üniversitesi Tıp Fakültesi Fiziksel Tıp ve Rehabilitasyon Anabilim Dalı, 20070 Kınıklı, Denizli, Turkey.

$$
\text { e-mail: fardic@pau.edu.tr }
$$


visceral fat has not been searched as a primary target widely. ${ }^{[7]}$ Among few studies, some authors ${ }^{[8]}$ reported a significant reduction in visceral adipose tissue without differences between the aerobic exercise intensity, suggesting no precious effect of the intensity, whereas the others ${ }^{[9]}$ reported the importance of aerobic exercise intensity in abdominal adiposity as a primary outcome parameter.

Walking is the easiest way to do physical activity among the choices of various physical activities and exercises. Achieving the moderate-intensity in an exercise program associated with a pedometer using steps per min (cadence) seems to be a promising method. Bouchard et al. ${ }^{[10]}$ suggested that the use of a pedometer to estimate the exercise intensity and reported that this method should be improved to the ability to estimate the moderate intensity.

In previous studies, the authors suggested solving this problem with general recommendations such as aerobic steps, step rate of 100 step per min, or 3000 steps per 30 min. ${ }^{[1-13]}$ However, the intensity of exercise is affected by the aerobic capacity of each individual. Therefore, the intensity should be managed individually in exercise prescription for physical activity. In a recent pilot study, we have demonstrated that the individualized number of steps can be achieved with our newly developed pedometer. ${ }^{[14]}$ In the literature, for the first time, we used this pedometer named as Equipment for Clever Exercise (ECE PEDO) reflecting the moderate intensity to investigate the efficacy of walking on abdominal obesity.

In the present study, we aimed to investigate the efficacy of both 12-week moderate aerobic walking with ECE PEDO and supervised treadmill walking on abdominal fat and to evaluate favorable changes in cardiorespiratory capacity in overweight and obese women.

\section{PATIENTS AND METHODS}

Sixty-five overweight or obese women who were admitted to the Department of Physical Medicine and Rehabilitation of Pamukkale University for obesity rehabilitation with the collaboration of Pamukkale University Sport Center were screened for eligibility between September 2014 and January 2015. Eligible participants who met the inclusion criteria were 26 to 60 years old women with a Body Mass Index (BMI) (in $\mathrm{kg} / \mathrm{m}^{2}$ ) from 27.1 to 39.6 and, waist circumference (WC) of $>80 \mathrm{~cm}$.
The exclusion criteria were as follows: BMI values less than $24.9 \mathrm{~kg} / \mathrm{m}^{2}$ and higher than $40 \mathrm{~kg} / \mathrm{m}^{2}$, the presence of an atherosclerotic heart disease, musculoskeletal or systemic diseases contradicting the exercise, a neurological or psychiatric disease affecting cooperation and cognitive function, and participation in a calorie restricted diets.

No diet was given to the participants during the study and they were informed about the continuation of their previous meal habits. The data were collected at an outpatient rehabilitation clinic in a tertiary university hospital. The study protocol, including the development of ECE PEDO and the investigation of its effectiveness in this publication, was approved by the Ethical Review Board of Pamukkale University (No. 04/2013), conducted according to standards established by the consort statement, and registered by ClinicalTrials.gov under No. NCT03109340. A written informed consent was obtained from each participant. The study was conducted in accordance with the principles of the Declaration of Helsinki.

The study was a randomized, single-center, twoarm, parallel-group, clinical study in overweight or obese women. The intervention took 12 weeks and all evaluations were performed at two time points: at baseline and at the end of the study. The intervention period was selected based on clinical trials which evaluated the effect of exercise on obesity. These studies showed that 12 weeks of exercise intervention was sufficient to cause benefits, as evidenced by the Cochrane review, assessing exercise as a means of achieving weight loss in individuals with overweight or obesity, using randomized-controlled clinical studies. ${ }^{[15]}$

Potential female adults were reviewed for eligibility and randomly allocated 1:1 into two groups: supervised treadmill group (Group 1, $\mathrm{n}=14$ ) or ECE PEDO pedometer group (Group 2, $\mathrm{n}=14$ ). The study staff allocated randomization numbers consecutively to the participants in the order that they attended the randomization visit.

\section{Assessment}

Anthropometric measurements: Weight and height were measured with weight scale Nan IB 150 and stadiometer. The BMI was calculated for as weight $(\mathrm{kg})$ divided by height squared $\left(\mathrm{m}^{2}\right)$. The WC was measured at the midpoint of the distance between the lowest rib and iliac crest with the non-elastic tape. ${ }^{[16]}$ All of the anthropometric measurements were performed and recorded by a single investigator. 
Abdominal visceral fat thickness (VFT) measurement: Ultrasonographic (USG) imaging was performed by the convex probe with $3.5 \mathrm{MHz}$ (General Electric Logic E9, USA). Abdominal VFT measurement was done with the placement of probe transversely at $1 \mathrm{~cm}$ above of the umbilicus, while the participant was lying in the supine position. ${ }^{[17]}$ The USG image was recorded after the expiration of the participant to prevent the effects of breathing and strain of abdominal wall on the measurements. The vertical line was marked on the frozen USG image between the front wall of the aorta and the inner surface of the rectus abdominal muscle for the measurement of abdominal VFT. The mean values of three measurements were recorded. ${ }^{[17]}$

Evaluation of cardiorespiratory capacity: The cardiopulmonary exercise test (CPET) was performed by gas exchange analysis system (CareFusion Germany $234 \mathrm{GmbH}$, Höchberg, Germany) on the treadmill (Technogym Excite Med, Cesena, Italy) with the modified Bruce protocol. The participants were monitored with the real-time, 12-lead electrocardiography during the test protocol. The heart rate (HR) was recorded during the exercise. The CPET was discontinued, when the respiratory exchange ratio $\geq 1.10$ and the $H R$ reached to $85 \%$ of max HR. ${ }^{[18]}$ The maximal oxygen consumption ( $\mathrm{VO}_{2} \mathrm{max}$ in milliliters per kilogram per min) reached at the end of exercise test was directly measured by ergospirometry analyzer was recorded.

The physical activity level: All participants before the exercise program was evaluated with the short form of the International Physical Activity Questionnaire (IPAQ-SF) which had the Turkish reliability and validity (Table 1). ${ }^{[19]}$ The IPAQ-SF consists of seven questions which give information about the time spent for sitting, walking, moderate activities, and severe activities for the last seven days.

\section{Interventions}

Exercise protocol: An exercise prescription was developed for each woman on the basis of the data acquired from the baseline treadmill exercise test. The target HR corresponding to values of 50 to $70 \%$ $\mathrm{VO}_{2} \max$ (moderate-intensity) were determined by the submaximal treadmill test.

During 12 weeks, five days in a week and $30 \mathrm{~min}$ per day walking program was applied to both groups. The participants were allowed non-attendance to the exercise program for maximum five sessions during 12 weeks.

1. Supervised treadmill group (Group 1): The participants were instructed walking at their target HR on the treadmill (Precor 932i) in Sports Rehabilitation Unit of Pamukkale University. HR was monitored by a Polar HR monitor (Polar Beat, Port Washington, NY). They did treadmill exercise for 30 min with the target HR corresponding to $50 \%$ of $\mathrm{VO}_{2} \max$ in first four weeks, and then $70 \%$ of $\mathrm{VO}_{2}$ max in following eight weeks.

2. ECE $P E D O^{\circ}$ pedometer group (Group 2): Following a 10-min rest after treadmill test, the participants were instructed to walk on the treadmill, while maintaining of their target HR. The number of steps taken in a min was calculated using a criterion pedometer Yamax SW200, when the target HR responses were reached. The procedure in described in detail in our previous work. ${ }^{[14]}$ In this way, we determined the steps per min required to moderate-intensity walking for each participant. Detected individual step numbers were recorded to the ECE PEDO for each participant. All participants were instructed 30 min brisk walking with the target step number corresponding the $50 \%$ of $\mathrm{VO}_{2}$ max for the first four weeks, and then $70 \%$ of $\mathrm{VO}_{2} \max$ for the following eight weeks.

Table 1. Baseline demographic and clinical characteristics of groups

\begin{tabular}{lcccc}
\hline & Group 1 $(\mathrm{n}=14)$ & & Group 2 $(\mathrm{n}=14)$ & \\
\cline { 2 - 2 } & Mean $\pm \mathrm{SD}$ & & Mean $\pm \mathrm{SD}$ & $p$ \\
\hline Age $($ year $)$ & $46.6 \pm 8.6$ & & $45.0 \pm 9.8$ & 0.765 \\
Height $(\mathrm{cm})$ & $158.6 \pm 6.4$ & & $156.9 \pm 6.2$ & 0.596 \\
Weight $(\mathrm{kg})$ & $83.3 \pm 12.2$ & & $78.8 \pm 11.1$ & 0.382 \\
Body Mass Index $\left(\mathrm{kg} / \mathrm{m}^{2}\right)$ & $33.0 \pm 3.5$ & $32.0 \pm 3.4$ & 0.435 \\
Waist circumference $(\mathrm{cm})$ & $97.6 \pm 7.2$ & $93.3 \pm 6.7$ & 0.093 \\
Hip circumference $(\mathrm{cm})$ & $114.9 \pm 8.1$ & $112.1 \pm 8.6$ & 0.408 \\
$\mathrm{VO}_{2}$ max $(\mathrm{mL} / \mathrm{min} / \mathrm{kg})$ & $21.4 \pm 2.7$ & $22.3 \pm 1.4$ & 0.448 \\
Visceral fat thickness $(\mathrm{cm})$ & $5.3 \pm 1.6$ & $4.8 \pm 1.2$ & 0.581 \\
\hline SD: Standard deviation; Group 1: Supervised treadmill group; Group 2: ECE PEDO pedometer group; $\mathrm{VO}_{2}$ max: Maximal \\
oxygen consumption.
\end{tabular}




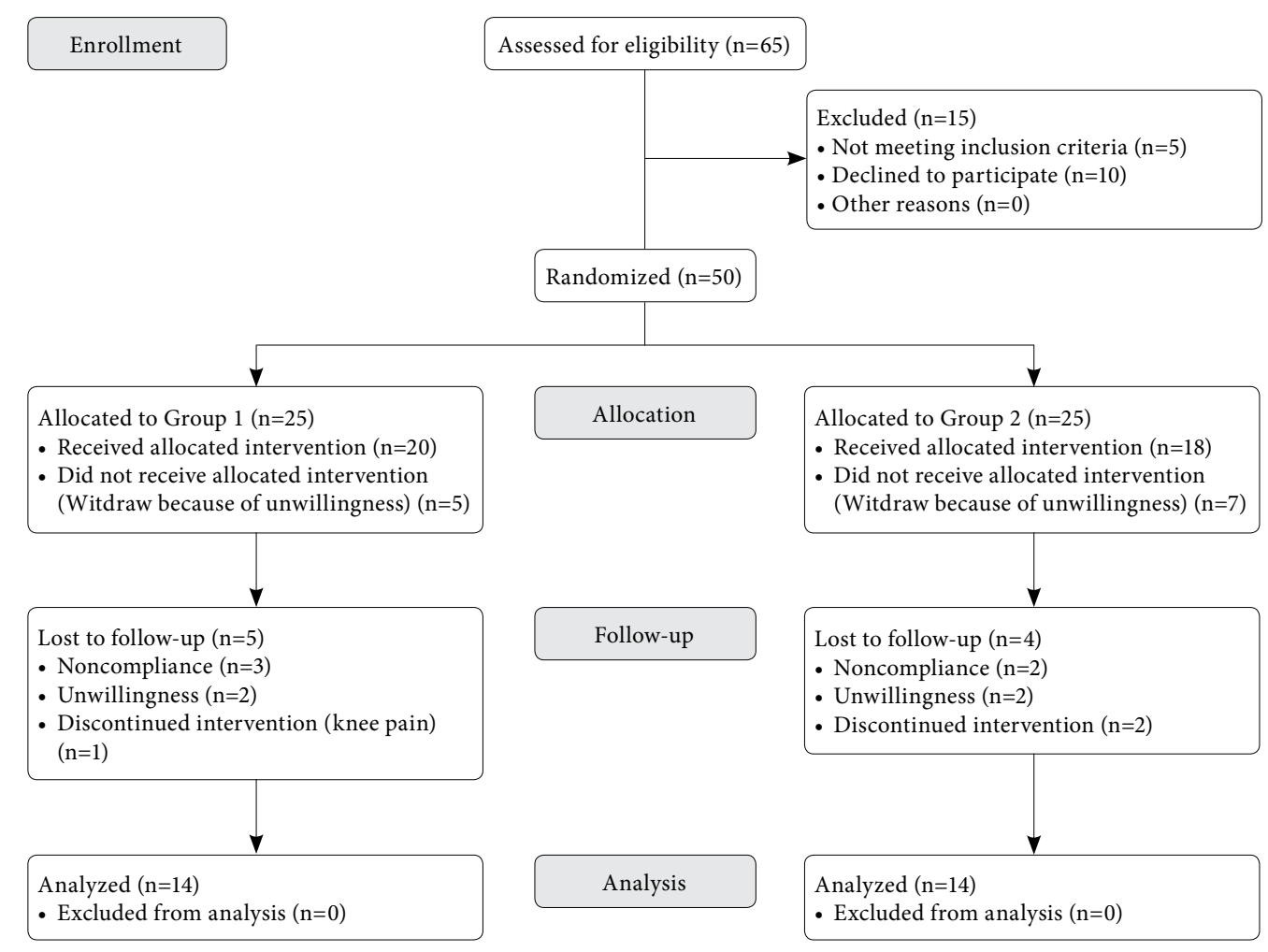

Figure 1. Participant flow through the study. Values are expressed as the number of participants. Group 1: Supervised treadmill group; Group 2: ECE PEDO pedometer group.

\section{Statistical analysis}

Statistical analysis was performed using the PASW version 17.0 software (SPSS, Inc., Chicago, IL, USA). Shapiro-Wilk test was used to determine the normality of the continuous variables. Descriptive statistics are expressed in mean \pm standard deviation (SD), frequency, and percentage. Baseline comparison of sociodemographic and clinical data between the groups was performed using the Student's $t$ test for the parametric quantitative data or Mann-Whitney $\mathrm{U}$ test for non-parametric quantitative data, and the Chi-square test was used to compare the qualitative data. Comparisons of pre- and post-treatment data of each group were done using the paired samples t-test for parametric data, or the Wilcoxon signed-rank test for non-parametric data. The comparison of treatment effectiveness between the groups was done using the Student's t-test for the parametric data or MannWhitney $U$ test for non-parametric data. The degree of association between the continuous variables were calculated using the Pearson's correlation coefficient. Linear regression analysis was used to determine the independent variables affected the difference of VFT of pre- and post-exercise program. A $p$ value of $<0.05$ was considered statistically significant.

Table 2. Comparison of the International Physical Activity Questionnaire - Short Form results between groups before aerobic exercise program

\begin{tabular}{|c|c|c|c|c|c|c|c|}
\hline \multirow[b]{2}{*}{ IPAQ-SF } & \multicolumn{3}{|c|}{ Group 1} & \multicolumn{3}{|c|}{ Group 2} & \multirow[b]{2}{*}{$p$} \\
\hline & Mean \pm SD & Median & Min-Max & Mean \pm SD & Median & Min-Max & \\
\hline Total Physical Activity Score (MET-min/week) & $585.7 \pm 282.8$ & 499.5 & $245-1164$ & $504.4 \pm 275.1$ & 480.5 & $139-1218$ & 0.550 \\
\hline Walking (MET-min/week) & $332.1 \pm 249.2$ & 297 & $0-924$ & $288.7 \pm 215.9$ & 198 & $99-792$ & 0.502 \\
\hline Moderate Physical Activity (MET-min/week) & $104.3 \pm 100.5$ & 100 & $0-240$ & $95.7 \pm 131.3$ & 50 & $0-400$ & 0.615 \\
\hline Severe Physical Activity (MET-min/week) & $151.4 \pm 256.0$ & 0 & $0-720$ & $120.0 \pm 266.2$ & 0 & $0-960$ & 0.678 \\
\hline Sitting (min) & $338.6 \pm 72.9$ & 360 & $180-480$ & $257.1 \pm 79.6$ & 240 & $120-420$ & $0.009^{*}$ \\
\hline
\end{tabular}


Table 3. Intragroup and intergroup comparisons of evaluation parameters between baseline and after exercise

\begin{tabular}{|c|c|c|}
\hline & Group $1(\mathrm{n}=14)$ & Group $2(n=14)$ \\
\hline & Mean \pm SD & Mean \pm SD \\
\hline \multicolumn{3}{|l|}{ Weight (kg) } \\
\hline Baseline & $83.3 \pm 12.2$ & $78.8 \pm 11.1$ \\
\hline After exercise & $82.0 \pm 13.1^{*}$ & $76.6 \pm 10.0^{*}$ \\
\hline \multicolumn{3}{|c|}{ Body Mass Index $\left(\mathrm{kg} / \mathrm{m}^{2}\right)$} \\
\hline Baseline & $33.0 \pm 3.5$ & $32.0 \pm 3.4$ \\
\hline After exercise & $32.5 \pm 3.7^{\star}$ & $31.1 \pm 3.3^{\star}$ \\
\hline \multicolumn{3}{|c|}{ Waist circumference $(\mathrm{cm})$} \\
\hline Baseline & $97.6 \pm 7.2$ & $93.3 \pm 6.7$ \\
\hline After exercise & $95.1 \pm 8.0^{*}$ & $89.9 \pm 6.7^{\star}$ \\
\hline \multicolumn{3}{|c|}{ Hip circumference $(\mathrm{cm})$} \\
\hline Baseline & $114.9 \pm 8.1$ & $112.1 \pm 8.6$ \\
\hline After exercise & $113.4 \pm 9.4^{*}$ & $110.9 \pm 7.9^{*}$ \\
\hline \multicolumn{3}{|c|}{$\mathrm{VO}_{2} \max (\mathrm{mL} / \mathrm{min} / \mathrm{kg})$} \\
\hline Baseline & $21.4 \pm 2.7$ & $22.3 \pm 1.4$ \\
\hline After exercise & $23.4 \pm 3.0^{\star}$ & $24.3 \pm 2.2^{\star}$ \\
\hline \multicolumn{3}{|c|}{ Visceral fat thickness $(\mathrm{cm})$} \\
\hline Baseline & $5.3 \pm 1.6$ & $4.8 \pm 1.2$ \\
\hline After exercise & $4.9 \pm 1.7^{\star}$ & $3.5 \pm 1.3^{*} \dagger$ \\
\hline
\end{tabular}

\section{RESULTS}

The participant flow through the study is depicted in Figure 1. Baseline demographic and clinical characteristics of both groups are shown in Table 1. There was no significant difference between the groups before the exercise program $(\mathrm{p}>0.05)$. No significant injury was observed in any of the groups.

When the groups were compared for the IPAQ-SF scores before the aerobic exercise program, there was no significant differences between the groups for per week scores of walking, moderate, severe, and total physical activity $(\mathrm{p}>0.05)$ (Table 2 ).

In-group and inter-group comparisons of the evaluation parameters between before and after exercise program are shown in Table 3. Significant decreases were found for weight, BMI, WC, and VFT after 12 weeks in both groups $(\mathrm{p}<0.05)$. Significant increases were observed for $\mathrm{VO}_{2}$ max after the exercise program in both groups $(\mathrm{p}<0.05)$.
Delta changes in the VFT were calculated using the difference between baseline and after measurements of the VFT (delta change in VFT=baseline VFT-VFT after exercise program) for both groups. Delta changes in the VFT were significantly better in Group 2 (Table 4).

Correlation analysis was performed to determine the relationships between the decrease of VFT and clinical variables. The change of VFT was calculated with the difference between the baseline VFT and the VFT of after exercise program. When the relationships between the change of VFT and changes of evaluation parameters were investigated, significant relationships were found between the weight loss and the decrease of VFT ( $p=0.039, r=0.393)$, and between the decrease of WC and decrease of VFT ( $\mathrm{p}=0.008, \mathrm{r}=0.488)$.

Linear regression analysis was performed to detect the independent variables affecting the decrease of VFT. The difference of weight, BMI, the difference of WC was included in the linear regression model as independent variables. The difference of WC was

Table 4. Comparison of delta change in visceral fat thickness between groups

\begin{tabular}{|c|c|c|c|c|c|c|c|}
\hline & \multicolumn{3}{|c|}{ Group 1} & \multicolumn{3}{|c|}{ Group 2} & \multirow[b]{2}{*}{$p$} \\
\hline & Mean $\pm S D$ & Median & Min-Max & Mean \pm SD & Median & Min-Max & \\
\hline Delta change in visceral fat thickness $(\mathrm{cm})$ & $0.36 \pm 0.69$ & 0.49 & $-1.63-1.43$ & $1.34 \pm 0.68$ & 1.45 & $-0.04-2.34$ & 0.001 \\
\hline
\end{tabular}


Table 5. Linear regression model for decrease of visceral fat thickness

\begin{tabular}{lccccc}
\hline & $\mathrm{B} \pm \mathrm{SE}$ & $\mathrm{Beta}$ & $\mathrm{t}$ & $p$ & Adjusted R $^{2}$ \\
\hline Difference of waist circumference & $0.142 \pm 0.050$ & 0.488 & 2.848 & 0.008 & \\
Difference of weight & $0.051 \pm 0.101$ & 0.130 & 0.508 & 0.616 & 0.238 \\
Body Mass Index & $-0.034 \pm 0.044$ & -0.137 & -0.770 & 0.449 & \\
\hline
\end{tabular}

SE: Standard error; Dependent variable, decrease of visceral fat thickness (VFT); Independent variables, difference of waist circumference (WC), difference of weight; Decrease of VFT: Baseline VFT-VFT after exercise program; Difference of weight: Baseline weight-weight after exercise program; Difference of WC: Baseline WC-WC after exercise program.

found as an independent variable which could predict the decrease of VFT, according to results of linear regression analysis $(\mathrm{p}=0.008)$ (Table 5).

\section{DISCUSSION}

To the best of our knowledge, this is the first randomized, prospective study to evaluate the effects of moderate-intensity walking with the novel pedometer in overweight or obese women. Our results showed that after a 12-weeks period, both walking groups showed significant improvements in anthropometric measurements, cardiorespiratory capacity, and VFT.

In our previous pilot study, we evaluated the capability of ECE PEDO to reflect the moderateintensity by the comparison of step counts with Yamax SW200 as the criterion pedometer. We showed high correlation and accordance between step counts of two pedometers in 30 min moderate aerobic exercise in women and men. ${ }^{[14]}$ On the basis of this, the present work is a secondary analysis of previously published work highlighting the importance of the feedback by ECE PEDO which guarantees to perform the moderate intensity.

The direct relationship between the exercise intensity and decreased the VFT has not yet been clarified. ${ }^{[7]}$ Gutin et al. ${ }^{[20]}$ reported no clear effect of the intensity of physical training on the reduction of visceral adiposity whereas Irving et al. ${ }^{[21]}$ reported the importance of exercise intensity in obese adults with abdominal visceral fat as a primary outcome parameter. On the other hand, Keating et al. ${ }^{[22]}$ reported a significant reduction in visceral adipose tissue in overweight/obese adults received different intensity aerobic exercise without differences between the intensity.

The ACSM recommends $30 \mathrm{~min}$ of moderateintensity aerobic exercise on most days of the week for individuals with overweight and obesity. The brisk walking is the easiest way to do it. Walking can be a useful recommendation for women with obesity or metabolic syndrome who have not had previous experience with exercise. ${ }^{[23]}$ Pedometers are commonly used in walking as a monitoring tool for physical activity. Current pedometers are useful indicators of daily step counting; however, cannot measure the intensity of physical activity and exercise. Therefore, it is difficult to establish whether the displayed step counts by the pedometer correspond to the required intensity of physical activity recommended by the guidelines. ${ }^{[24]}$ However, Rice et al., ${ }^{[25]}$ showed that many individuals are unable to determine their intensity of physical activity and exercise. This brings a challenging problem, as health profits are related to the intensity of activity. ${ }^{[12]}$ Although current pedometers do not, is it possible to translate current recommendations for the intensity of physical activity into a pedometerbased exercise? ${ }^{[12]}$ Our new pedometer records timestamped step goals range, unlike simple pedometers. Individualized lower and upper limits of target step numbers per min obtained from baseline treadmill test are uploaded to the ECE PEDO for each individual. The ECE PEDO gives audial feedback to the participant instantly according to these uploaded targets and displays a personal report about the step counts in certain durations reflecting the intensity of walking. In the present study, we showed that 12-week walking exercise with the sufficient moderateintensity provided with the association between the HR and pedometer step counts resulted in the decrease in abdominal obesity. ${ }^{[14]}$

Recently, Hong et al. ${ }^{[26]}$ investigated the effect of 12 -week walking exercise on abdominal fat in obese women. The walking exercise was performed at the exercise intensity as 50 to $60 \%$ of $\mathrm{VO}_{2} \max$. Whether the HR during the walking exercise reached the target HR was confirmed using the Polar heart monitor. ${ }^{[26]}$ They found that the exercise led to significant reductions in both subcutaneous and visceral adiposities in consistent with our study. We believe that using a pedometer giving instant personalized audible feedback is simpler and user-friendly approach. The ECE PEDO sets the tempo by giving audible warnings to individuals to enable them to adjust brisk walking speed allowing them to determine the exercise intensity easily. 
There are some reports in the literature about the required number of steps to reach the recommendation of moderate-intensity physical activity. The majority of pedometer studies recommend daily step goals in walking. ${ }^{[23]}$ The most widely noticed step recommendation is to achieve 10,000 steps per day, although this goal does not express the physical activity intensity. ${ }^{[23]}$ Le Masurier et al. ${ }^{[27]}$ revealed that achieving 10,000 steps a day does not guarantee to correspond the guidelines to provide the health benefits of physical activity. Recent studies ${ }^{[11,23]}$ have shown that moderate-intensity walking appears approximately equal to at least $100 \mathrm{step} \cdot \mathrm{min}-1$. Since fixed step counts per min is a poor indicator for $\mathrm{VO}_{2} \max$, the same recommendations are not suitable for all persons. All of these authors have suggested using a fixed value for everybody to determine the intensity. Unlike these approaches, in the present study, individualized moderate activity intensity and related pedometer step counts were calculated. Determining the individualized intensity of physical activity by a pedometer and its efficacy has not been demonstrated previously in the literature. Goal setting and activity monitoring were demonstrated as effective self-management strategies to improve exercise adherence. ${ }^{[28]}$ In our study, the step range per min feedback was used as an intensive and objective monitoring tool.

In addition to the determination of the required step numbers for the moderate-intensity walking, individual differences of aerobic capacities of individuals should be kept in mind. Individuals with low fitness need lower training intensities than individuals with higher baseline fitness. Lubans et al. ${ }^{[28]}$ showed the relationship between step counts and exercise intensity, as measured by the HR in adolescents. In their method, total steps taken on pedometer treadmill trial for $10 \mathrm{~min}$ were divided by 10 to find a prediction of steps per min. They recommended that the targeted number of steps should be managed according to the existing fitness levels, due to less fit adolescents taking the lower number of steps than fitter adolescents. In our study, we measured the personal aerobic capacity to determine step count targets according to existing fitness levels. Conferring target step counts, the ECE PEDO provided instructions to the participants to walk the calculated step range corresponding to the target $\mathrm{HR}$.

Assessing body fat distribution and in tracking changes in body composition over exercise training programs in individuals with obesity has been still conducted. Monitoring of body composition is applicable to individuals with obesity using various methods and equipment. One of the imaging methods that determine fat distribution is USG which is a reliable and convenient way of quantifying the amount of visceral fat. ${ }^{[17]}$ It has become a used method to evaluate the fat thickness and distribution, as besides being reliable it is cheaper and widespread than the computed tomography (CT) and magnetic resonance imaging (MRI) measurements and does not have harmful effects such as radiation. ${ }^{[29]}$ In this study, USG measurements showed that the both walking exercise groups result in the decrease of VFT after the 12 weeks. The difference of WC was detected as an independent variable which predicts the decrease of VFT. The decrease in WC is particularly important, as this parameter is considered to be an independent factor of cardiovascular risk. ${ }^{[30]}$

Limitations of this study include the relatively small number of participants completing the intervention and short follow-up. As the ECE PEDO is a practical, simple to use and wearable activity monitor that is feasible for use in clinical and research settings, further studies should investigate the utility of ECE PEDO in different intensity walking and explore the long-term effectiveness in different populations.

In conclusion, our study shows that a 12-week moderate-intensity walking both with ECE PEDO's feedback and supervised treadmill walking reduce abdominal fat in overweight and obese women. Thus, individualized moderate-intensity physical activity with the ECE PEDO would be able to do in anywhere for gaining the health benefit.

\section{Declaration of conflicting interests}

The authors declared no conflicts of interest with respect to the authorship and/or publication of this article.

\section{Funding}

This work was supported by Pamukkale University Scientific Research Fund with Project Number: 2013TPF009.

\section{REFERENCES}

1. World Health Organization. Obesity and Overweight. Global Strategy on Diet. Physical Activity and Health. Fact Sheet; 2006, No 311.

2. Haskell WL, Lee IM, Pate RR, Powell KE, Blair SN, Franklin BA, et al. Physical activity and public health: updated recommendation for adults from the American College of Sports Medicine and the American Heart Association. Med Sci Sports Exerc 2007;39:1423-34.

3. Oh S, Tanaka K, Noh JW, So R, Tsujimoto T, Sasai H, et al. Abdominal obesity: causal factor or simply a symptom of obesity-related health risk. Diabetes Metab Syndr Obes 2014;7:289-96. 
4. Ismail I, Keating SE, Baker MK, Johnson NA. A systematic review and meta-analysis of the effect of aerobic vs. resistance exercise training on visceral fat. Obes Rev 2012;13:68-91.

5. Durstine JL, Moore GE, Painter PL, Macko R, Gordon BT, Kraus WE. Chronic conditions stongly associated with physical inactivity. In: Moore GE, Durstine JL, Painter PL, editors. ACSM's Exercise management for persons with chronic diseases and disabilities. 4th ed. Champaign, IL: Human Kinetics; 2016. p. 80-4.

6. U.S. Department of Health and Human Services. Physical Activity Guidelines for Americans. 2008, ODPHP Publication No. U0036. Available from: www.health.gov/ paguidelines.

7. Vissers D, Hens W, Taeymans J, Baeyens JP, Poortmans J, Van Gaal L. The effect of exercise on visceral adipose tissue in overweight adults: a systematic review and meta-analysis. PLoS One 2013;8:e56415.

8. Keating SE, Hackett DA, Parker HM, O’Connor HT, Gerofi JA, Sainsbury A, et al. Effect of aerobic exercise training dose on liver fat and visceral adiposity. J Hepatol 2015;63:174-82.

9. Irving BA, Davis CK, Brock DW, Weltman JY, Swift D, Barrett EJ, et al. Effect of exercise training intensity on abdominal visceral fat and body composition. Med Sci Sports Exerc 2008;40:1863-72.

10. Bouchard DR, Langlois MF, Boisvert-Vigneault K, Farand P, Paulin M, Baillargeon JP. Pilot study: can older inactive adults learn how to reach the required intensity of physical activity guideline? Clin Interv Aging 2013;8:501-8.

11. Abel M, Hannon J, Mullineaux D, Beighle A. Determination of step rate thresholds corresponding to physical activity intensity classifications in adults. J Phys Act Health 2011;8:45-51.

12. Marshall SJ, Levy SS, Tudor-Locke CE, Kolkhorst FW, Wooten KM, Ji M, et al. Translating physical activity recommendations into a pedometer-based step goal: 3000 steps in 30 minutes. Am J Prev Med 2009;36:410-5.

13. Tudor-Locke C, Hatano Y, Pangrazi RP, Kang M. Revisiting "how many steps are enough?".Med Sci Sports Exerc 2008;40:537-43.

14. Ardic F, Göcer E. Cadence Feedback With ECE PEDO to Monitor Physical Activity Intensity: A Pilot Study. Medicine (Baltimore) 2016;95:3025.

15. Shaw K, Gennat H, O'Rourke P, Del Mar C. Exercise for overweight or obesity. Cochrane Database Syst Rev 2006;4:CD003817.

16. Ardic F. Anthropometry and exercise in obesity. In; Preedy VR, editor. Handbook of Anthropometry: Physical Measures of Human Form in Health and Disease. New York: Springer; 2012. p. 1919-35.

17. Oh J, Kim SK, Shin DK, Park KS, Park SW, Cho YW. A simple ultrasound correlate of visceral fat. Ultrasound Med Biol 2011;37:1444-51.
18. Neves CD, Lacerda AC, Lage VK, Lima LP, Fonseca SF, de Avelar NC, et al. Cardiorespiratory responses and prediction of peak oxygen uptake during the shuttle walking test in healthy sedentary adult men. PLoS One 2015;10:e0117563.

19. Saglam M, Arikan H, Savci S, Inal-Ince D, Bosnak-Guclu M, Karabulut E, et al. International physical activity questionnaire: reliability and validity of the Turkish version. Percept Mot Skills 2010;111:278-84.

20. Gutin B, Barbeau P, Owens S, Lemmon CR, Bauman M, Allison J, et al. Effects of exercise intensity on cardiovascular fitness, total body composition, and visceral adiposity of obese adolescents. Am J Clin Nutr 2002;75:818-26.

21. Irving BA, Davis CK, Brock DW, Weltman JY, Swift D, Barrett EJ, et al. Effect of exercise training intensity on abdominal visceral fat and body composition. Med Sci Sports Exerc 2008;40:1863-72.

22. Keating SE, Hackett DA, Parker HM, O'Connor HT, Gerofi JA, Sainsbury A, et al. Effect of aerobic exercise training dose on liver fat and visceral adiposity. J Hepatol 2015;63:174-82.

23. Tudor-Locke C, Craig CL, Brown WJ, Clemes SA, De Cocker K, Giles-Corti B, et al. How many steps/day are enough? For adults. Int J Behav Nutr Phys Act 2011;8:79.

24. Welk GJ, Differding JA, Thompson RW, Blair SN, Dziura J, Hart P. The utility of the Digi-walker step counter to assess daily physical activity patterns. Med Sci Sports Exerc 2000;32:481-8.

25. Rice KR, Heesch KC, Dinger MK, Fields DA. Rice KR, Heesch KC, Dinger MK, Fields DA. J Phys Act Health 2008;5:58-73.

26. Hong HR, Jeong JO, Kong JY, Lee SH, Yang SH, Ha CD, et al. Effect of walking exercise on abdominal fat, insulin resistance and serum cytokines in obese women. J Exerc Nutrition Biochem 2014;18:277-85.

27. Le Masurier GC, Sidman CL, Corbin CB. Accumulating 10,000 steps: does this meet current physical activity guidelines? Res Q Exerc Sport 2003;74:389-94.

28. Lubans DR, Morgan PJ, Collins CE, Boreham CA, Callister R. The relationship between heart rate intensity and pedometer step counts in adolescents. J Sports Sci 2009;27:591-7.

29. Hirooka M, Kumagi T, Kurose K, Nakanishi S, Michitaka K, Matsuura B, et al. A technique for the measurement of visceral fat by ultrasonography: comparison of measurements by ultrasonography and computed tomography. Intern Med 2005;44:794-9.

30. Skrypnik D, Bogdański P, Mądry E, Karolkiewicz J, Ratajczak M, Kryściak J, et al. Effects of Endurance and Endurance Strength Training on Body Composition and Physical Capacity in Women with Abdominal Obesity. Obes Facts 2015;8:175-87. 